\title{
Crisis económicas, innovación y reglas monetarias
}

\author{
Juan E. Castañeda Fernández \\ Departamento de Economía Aplicada y Gestión Pública. \\ Facultad de CC.EE. y EE. (UNED). jcastanedaf@cee.uned.es

\section{Félix F. Muñoz Pérez} \\ Departamento de Análisis Económico: \\ Teoría Económica e Historia Económica. \\ Facultad de CC.EE. y EE. (UAM).felix.munoz@uam.es
}

\section{INTRODUCCIÓN}

En este artículo partimos de que las crisis recurrentes del sistema económico no obedecen a factores únicamente reales o puramente monetarios, sino a ambos. Defendemos que las características del sistema financiero actual y, en particular, las reglas monetarias que siguen los bancos centrales, inciden sobre los procesos «reales» que se generan en los mercados exacerbando las fases expansivas, alimentando «monetariamente» las burbujas especulativas, y con ello agravando posteriormente las consecuencias de las contracciones, lo que en definitiva aumenta la virulencia de las crisis. Así, diremos que (1) las crisis tienen un origen real en las economías de mercado, relacionado con la dinámica de generación de expectativas asociadas a los procesos de innovación e inversión, ya que una parte importante de las mismas devienen irrealizables con el paso del tiempo; y, asimismo (2) las características del sistema de generación de liquidez actual permiten, precisamente durante las etapas expansivas, el desarrollo de nuevos procesos de inversión sobre bases monetarias y condiciones de crédito insostenibles a medio y largo plazo.

Por supuesto, la explicación propuesta no agota el tema tan rico y complicado de la dinámica evolutiva de las economías de mercado mediante movimientos expansivos y contractivos recurrentes, pero sí señala claramente que la naturaleza de éste movi- 
miento no es puramente monetaria; al menos, la causa que lo desencadena no tiene porqué serlo como regla general. En nuestra opinión, la causa hay que buscarla en el dinamismo que el comportamiento empresarial despliega, a través de los procesos de innovación y en las oleadas de novedades que se producen en el sistema económico. El sistema financiero actúa sobre esta dinámica empresarial a través de una de sus principales funciones: la provisión de liquidez y crédito a los agentes económicos. Cómo desempeña esta función y su influencia en las crisis es uno de los principales puntos a tratar en este trabajo. No creemos que sea la configuración actual del sistema financiero lo que provoque de suyo la sucesión de movimientos expansivos y contractivos; pero sí que la forma de gobierno actual de la liquidez en dicho sistema no es neutral: puede tener un efecto decisivo en la prolongación temporal y en la magnitud de tales oscilaciones de la economía.

El artículo está organizado como sigue. En el epígrafe 2 se presenta esquemáticamente cómo los movimientos expansivos y contractivos de una economía dinámica se basan en el comportamiento de empresarios «schumpeterianos» y en el papel del crédito en ese proceso. A continuación, se expondrá en el epígrafe 3 el papel que el sistema financiero $y$, especialmente, los bancos centrales, desempeñan en el agravamiento de los movimientos característicos de estos procesos. El sistema financiero-institucional, que es resultado de un proceso evolutivo específico (Smith, 1936), constituye uno de los elementos fundamentales del actual paradigma técnico-económico. Nos referimos en particular a uno de sus elementos, el uso de reglas monetarias por parte de los bancos centrales, que implícita o explícitamente tienen por objeto «estabilizar la economía»a través del control de la tasa de inflación; lo que puede producir el efecto paradójico de agravar las depresiones a través de una sobre-emisión de liquidez durante las expansiones. En el epígrafe 4 se presentan unas breves conclusiones así como una propuesta de investigación futura en la línea apuntada en este trabajo.

\section{EMPRESARIALIDAD, EXPANSIÓN CREDITICIA Y CRISIS}

\subsection{Algunas clarificaciones previas}

Una crisis económica es el paso de una fase expansiva de la economía a otra contractiva, dentro de un movimiento mucho más general, de crecimiento secular. En la medida en que un sistema económico evoluciona conforme al esquema: fase de expansión, crisis, contracción, nueva fase de expansión, etc., debe colegirse que las causas económicas de la fase contractiva, y por tanto de la propia crisis, han de buscarse 
en la dinámica específica de la fase expansiva. ${ }^{1}$ Por este motivo, en este trabajo nos concentramos en estudiar la interrelación entre las causas reales y monetarias que se desarrollan precisamente en los movimientos expansivos como medio para estudiar las causas últimas de las crisis. El examen de las causas que gobiernan los movimientos económicos de largo plazo, la coyuntura económica secular (Dupriez, 1959), es un trabajo bien complicado, si bien de la máxima importancia tanto teórica como práctica, como manifiesta el debate sobre la adopción de medidas de política económica ante la actual crisis financiera y económica internacional.

Preferimos el término «movimiento económico»o «coyuntura económica» en lugar del más habitual «ciclo económico» porque éste último término implica, o al menos sugiere, el carácter cíclico de los movimientos económicos, lo cual presupone dos cosas: una cuasi-autonomía de los procesos económicos que hace que éstos, de forma automática, desplieguen fases sucesivas de expansión-contracción; y la naturaleza homogénea de los mismos (piénsese, por ejemplo, en un movimiento oscilatorio simple). Sin embargo, la evolución de un sistema económico se caracteriza no sólo porque ese sistema experimenta cambios en las magnitudes que lo describen, sino fundamentalmente cambios cualitativos. Cosa bien distinta es el carácter recurrente de la sucesión de fases de expansión y recesión; pero en cada caso, en cada nueva fase, nuevos datos (nuevas tecnologías, formas institucionales etc.) están apareciendo (y desapareciendo) en un proceso en el que la dinámica de transformación del sistema tiende a romper las inercias hacia los estados de equilibrio estacionario implicados por los datos económicos existentes en cada momento del tiempo; buscando, sin embargo, una relación coherente a largo plazo entre los nuevos elementos que van surgiendo en interacción en el sistema (trayectorias tecnológicas, instituciones, etc.). Una verdadera «teoría del ciclo» debería reconocer la posibilidad de que se presenten novedades esenciales (Shackle, 1972: 340).

Sin embargo, después de la Segunda Guerra Mundial, el tipo de análisis más convencional de la dinámica de la economía no ha procedido, por lo general, de este modo. Habitualmente se ha planteado el análisis de la aparición recurrente de las crisis económicas en el sistema capitalista bajo la forma de una pregunta sobre su carácter real o monetario, jasumiendo el carácter exógeno de la tecnología y las preferencias! Responder a esta cuestión sería algo de la mayor importancia si no fuese porque, a nuestro juicio, se trata de una pregunta mal planteada. En la base de la pregunta se encuentra

${ }^{1}$ Pueden existir otras causas extraeconómicas como, por ejemplo, una invasión extranjera que dé lugar a una guerra, que produzcan movimientos de este tipo. Las semillas de la posterior recuperación deberán encontrarse, lógicamente, en la dinámica propia de la contracción. 
una falsa dicotomía en lo que constituye un único fenómeno económico: el proceso de cambio o de evolución en una economía de mercado que, eso sí, presenta aspectos tanto «reales» como monetarios. Que se trata de una pregunta mal planteada es fácil de comprobar en las pseudo-explicaciones de los «ciclos económicos» que se han dado mayoritariamente. Por ejemplo, los monetaristas, si bien logran exponer muy detalladamente los mecanismos de transmisión de una expansión del crédito en los precios de los activos y de los bienes y servicios, no aclaran cuáles son las causas últimas que inician las crisis (más allá de una vaga apelación a la falta de oportunidades de inversión, la liquidación de malas inversiones, los efectos derivados de la contracción del crédito en un momento dado, etc.). Buenos ejemplos de este tipo de explicaciones son Fisher (1933) y Friedman y Schwartz (1963). Por su parte, los economistas que defienden que el ciclo económico es fundamentalmente un fenómeno real (véase Galí, 2008) no tienen en cuenta, al menos suficientemente, la naturaleza tan especial de los fenómenos e instituciones monetarias y los efectos «reales» que su funcionamiento producen.

En este estado de cosas parece absolutamente necesario una teoría que integre en un único modelo explicativo tanto las causas «reales» como las financieras, a fin de dar cumplida explicación de este particular modo de expansión económica que es propio del sistema capitalista moderno. En este sentido, cabe destacar los trabajos de dos de los mayores representantes de la llamada Escuela Austríaca: Mises y Hayek. Mises (1924, 1949), en el que es quizá el intento más completo de integrar ambas esferas económicas (la real y la monetaria), construye su teoría monetaria y del crédito sobre la misma base explicativa (la teoría subjetiva del valor) con que analiza los procesos de mercado del resto de bienes y servicios de la economía. En la misma línea, la obra de Hayek (1931), no siempre bien comprendida (Hicks, 1967), busca una relación entre el dinero, los precios y la producción a través del estudio del origen y expansión de la estructura de capital de una economía (véase su interpretación moderna en Garrison, 2005).

En este trabajo buscamos, al menos parcialmente, una integración de ambos ámbitos de lo económico; pero, en lo que a los factores reales se refiere, emplearemos una aproximación diferente a la de los autores anteriormente señalados. No nos basaremos en una teoría del valor (objetivo fuera del alcance de este artículo), sino en una que describe el comportamiento de los agentes, en particular de los empresarios y de los banqueros. ${ }^{2}$ Se trata de una aproximación muy cercana a la tradición «schumpeteriana»

2 Evidentemente, esta aproximación no agota ni de lejos una explicación más substantiva de los movimientos económicos; muy al contrario: como han puesto de manifiesto Encinar y Muñoz (2006), la teoría «schumpeteriana» adolece de una deficiencia en su fundamentación económica 
y que ha sido recientemente revitalizada tanto en la corriente de la llamada economía evolutiva como en la literatura del cambio económico y del progreso tecnológico. Sirvan de ejemplo, las obras de Nelson y Winter (1982), Pérez (2004) y Freeman y Louça (2001), entre otros muchos.

\subsection{Las causas «reales» de las crisis: una primera aproximación ${ }^{3}$}

La economía capitalista no evoluciona de forma armónica o suave, sino a través de procesos o movimientos recurrentes de expansión-contracción de la actividad económica, que se han denominado «ciclos económicos». ${ }^{4} \mathrm{El}$ mecanismo que impulsa estos movimientos es la acumulación (y estructura) de capital. La acumulación de capital

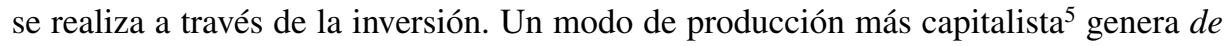
suyo una dinámica de interacción temporal entre inversión y consumo (y ahorro). Una explicación de cómo surgen las expansiones y recesiones sería como sigue. Si definimos la inversión como la producción de mejoras en las circunstancias de la sociedad en el futuro, aumentar la inversión implicará que la economía rinde menos servicios para el disfrute de necesidades inmediatas a cambio de la expectativa de recibir más en el futuro. Ahora bien, los agentes que desarrollan la actividad innovadora e inversora, los empresarios, reciben y gastan parte de las rentas generadas por los procesos de inversión en forma de aumento de su consumo presente de bienes y servicios. De este modo, además de su expectativa de disfrutar de más bienes en el futuro, la demanda presente de bienes de consumo se verá aumentada (de un modo quizá no totalmente descontado) como consecuencia de un cierto «efecto riqueza» asociado al aumento en la cotización de los activos entre aquellos que han ordenado las inversiones. Atendiendo a la dinámica de las inversiones y a su extensión temporal, este aumento de la demanda de consumo presente supone un freno a la extensión de los procesos de inversión; ya que parte

última, precisamente en su pieza clave, la teoría de la innovación. Sin embargo, nuestra elección obedece aquí a una estrategia expositiva que trata de iluminar aspectos clave para la explicación de las crisis recurrentes.

${ }^{3}$ En este apartado seguimos a Shackle (1972) en su explicación de un «ciclo epistémico».

${ }^{4}$ Matsuyama (1999); y en otro orden Pérez (2004).

5 Entendemos por economía capitalista la que basa su crecimiento en la extensión de métodos de producción más indirectos —es decir, más intensivos en capital. En las economías occidentales, este tipo de sistema de producción tiende a organizarse alrededor de mercados más o menos libres. 
de los recursos productivos de la sociedad tienen que destinarse a satisfacer esta necesidad presente de disponer de más bienes y servicios. Si no aumenta la tasa de ahorro, y en ausencia de medidas monetarias que distorsionen esta información «real», esto provoca que no haya más recursos para expandir aún más los procesos productivos. Es en este punto donde las particulares características del sistema de creación actual de liquidez entran en juego, como veremos en el epígrafe 3.

Si esta fase expansiva está apoyada por un crecimiento del crédito, el desarrollo de los procesos de innovación e inversión empresarial permitirá un aumento del conjunto de bienes y servicios presentes, así como la expectativa de su aumento en el futuro. Este proceso acumulativo puede generar una creencia en parte o en la totalidad de los empresarios (y en parte o en la totalidad de los consumidores) en la indefinida pervivencia de estos síntomas continuamente «alcistas»; a saber, el progresivo incremento en las cantidades físicas de bienes vendidos por unidad de tiempo y el progresivo incremento de sus precios.

Tendríamos aquí el origen de un auge (fase expansiva de un movimiento) económico. ¿Cómo se produciría el paso a una fase contractiva? La convulsión general en las mentes de los hombres de negocio debida a tal creencia en la continuidad de la expansión dará lugar a un empujón extra, pero ya último, al flujo de inversión. A partir de ese momento el fortalecimiento de la demanda general que surge de esta última aceleración en la senda de gasto para aumentar y mejorar los equipos habrá sido esperado. La inercia del proceso acumulativo no estará ya vinculada a mejoramientos adicionales, sino que quedará vinculada a la confirmación de la prospectiva (expectativa) de los negocios ${ }^{6}$. El incentivo para incrementar aún más el flujo de inversiones comenzará a faltar. Lo mismo, por tanto, sucederá con la actual anticipación del fortalecimiento de la demanda: las expectativas de una continua expansión de la economía quedarán ya defraudadas y los créditos, dado el más que probable incremento en el nivel de endeudamiento de los agentes, no podrán reintegrarse en su totalidad. Un sentimiento de agotamiento comenzará a extenderse por todos los sectores, dando así lugar a la crisis. El incentivo a invertir será menor, lo que conducirá a un debilitamiento de la demanda general, también, quizá, contrario a la expectativa. Llegados a este punto se puede generar un proceso de des-acumulación. Una secuencia posterior de re-evaluaciones por

${ }^{6}$ Un ejemplo: antes de que estalle una burbuja bursátil, ya nadie espera que las empresas vayan a valer más, pero a pesar de esto sigue habiendo, probablemente, una posibilidad de generar una plusvalía adicional por la inercia del alza de la bolsa (siempre y cuando uno sea el penúltimo en vender). 
parte de los hombres de negocio, podrá, eventualmente, parar este proceso volviendo la economía a la senda de la recuperación.

\subsection{El papel de las expectativas}

Estas interacciones entre inversión y consumo pueden producirse en varios niveles y horizontes temporales. Pueden producirse en un corto, medio o largo plazo, y obedecer a causas de corto y medio plazo $^{7}$, o bien a factores seculares vinculados, de forma genérica, al progreso técnico. Todo esto daría lugar a ondas de corto, medio o largo plazo (ciclos Kittchin, Juglar o Kondratieff, respectivamente). En cualquier caso, es importante señalar el papel de las expectativas, esto es, del conocimiento de los agentes, en la explicación de estas fluctuaciones.

Si nos centramos en estos últimos, en los factores seculares, podemos afirmar a la luz de la experiencia histórica que abarca desde la Revolución Industrial hasta nuestros días, sin temor a equivocarnos, que el origen de estos movimientos suele estar ligado, en la fase inicial de un movimiento expansivo secular, a la aparición de innovaciones. Schumpeter $(1934 ; 1939)$ buscó la causa última de las fluctuaciones económicas (y, en definitiva, del desenvolvimiento económico) en los procesos de innovación iniciados por los agentes del cambio económico: los empresarios ${ }^{8}$. Según este tipo de explicaciones, la nota característica de la economía capitalista es que «progresa» a base de innovaciones -tanto técnicas como organizativas- introducidas por empresarios que se financian con la expansión del crédito. Dadas la naturaleza y dinámica de dichas innovaciones, éstas tienen un carácter acumulativo y complementario, que habitualmente no se presentan independientemente unas de otras, sino formando clusters de innovaciones (en el tiempo y en el espacio). Cuando un cluster de innovaciones irrumpe en el sistema económico, da lugar a una «revolución tecnológica» o «nueva economía», que trae aparejada grandes expectativas de ganancia vinculadas a esas nuevas tecnologías, generando una fase de expansión de la actividad que usualmente degenera en burbujas especulativas.

7 Por ejemplo un ciclo político.

${ }^{8}$ En rigor «empresarios schumpeterianos», dada la diferenciación estricta que establece Schumpeter entre los empresarios en cuanto agentes que desempeñan una función muy especial (introducir novedades en el sistema económico) y los meros directores de empresa. 
Cuando una nueva «revolución tecnológica» irrumpe, no sólo se añaden algunas industrias nuevas y dinámicas a la estructura productiva previa. Mediante la configuración de un paradigma tecno-económico (Dosi, 1982) nuevo, se proporcionan los medios para modernizar todas las industrias y actividades existentes. El proceso de difusión tanto de la revolución como de su paradigma a todo lo largo de la economía constituye una gran oleada de desarrollo.

Partiendo de una economía que tiende a un estado estacionario por agotamiento de un paradigma tecno-económico previo, podemos aproximarnos al inicio de una fase expansiva de la siguiente manera: se crean expectativas de fuerte crecimiento en torno al potencial esperado de nuevas innovaciones. Cada oleada de innovaciones consta de dos períodos principales: (a) el período de instalación, que es turbulento y en que se manifiestan todas las tensiones que entraña el nuevo paradigma en su lucha con el antiguo, y (b) el período de despliegue del mismo, en el que una vez triunfante, el nuevo paradigma tecno-económico despliega toda su lógica en un entorno de ganancia de coherencia socio-institucional.

Siguiendo a Pérez (2004), el primer período se rige, en su implantación, por el capital financiero, que toma la iniciativa en el impulso de los nuevos sectores que incorporan el nuevo paradigma. En este caso son los empresarios «schumpeterianos» los que deben lograr convencer a los banqueros para que financien sus innovaciones a través de la expansión del crédito. El agente de la innovación es el empresario, no el capitalista, que no corre con los riesgos de sus posibles errores empresariales (que dan lugar a «malas inversiones»). Sin embargo, para poder llevar a cabo sus innovaciones requiere redirigir recursos de la economía. Esto implica el acceso al crédito, crédito que no es necesariamente un ahorro acumulado9. Las nuevas inversiones asociadas a la oleada de innovaciones generan una dinámica como la descrita en el apartado 2.2 anterior.

Aquí el papel de los bancos es fundamental: son estos quienes guían la expansión del crédito a través de la posibilidad de crear medios de pago ex novo. En un sistema fiduciario de reserva fraccionaria y con un banco central que asume el papel de prestamista de última instancia, se plantea el problema del límite a esta expansión de ese crédito. En principio, son los bancos quienes asumen los «riesgos» vinculados a esas innovaciones, poniendo en peligro incluso su propia solvencia (posiblemente derivada de problemas de liquidez, pero no sólo). Pero en la medida en que existe un agente que garantiza la liquidez de todo el sistema, el riesgo se «reparte» en toda

${ }^{9}$ Véase especialmente Schumpeter (1934), capítulo III. 
la economía. Así, los bancos expanden el crédito emitiendo nuevos instrumentos de pago; es decir, expandiendo un crédito generado ex novo. Asumiendo que el proceso se desenvuelva en un marco financiero-institucional como el actual, el problema no es tanto crear medios de pago in vacuo, como que estos fracasen en el objetivo para el que fueron creados; y esto es lo que sucede cuando surgen las dificultades para el afianzamiento de las nuevas tecnologías (o, en un sentido más general, del nuevo paradigma tecno-económico $)^{10}$. La expansión del crédito es la otra cara de la innovación.

La fase expansiva se detiene y cambia a contractiva cuando se esfuman las ventajas que inicialmente asociaron los agentes a las innovaciones. Para ese momento ciertas inversiones no pueden llevarse a efecto y deben liquidarse con pérdida. Pronto se ve que las expectativas de grandes ganancias eran en buena medida infundadas, las tasas de beneficios inicialmente previstas se revisan y caen y la devolución de los créditos se hace muy difícil ${ }^{11}$.

En el segundo período es el capital productivo el que domina: los estándares de las nuevas tecnologías se afianzan, las instituciones se ajustan a las nuevas necesidades de los negocios, etc. Es el momento del «director de empresa schumpeteriano». El carácter de las innovaciones tiene ahora más que ver con el acomodo y perfeccionamiento de la tecnología genérica que con la introducción de innovaciones tecnológicas radicales. En ambos casos, tanto en la fase (a) como en la (b), las innovaciones imponen una reorientación hacia nuevos equilibrios asociados a la absorción de esas nuevas tecnologías: efectos de absorción, exploración de nuevas oportunidades de negocio, difusión por toda la economía, etc. Lo nuevo se afianza y despliega toda su potencialidad.

Pero entre la fase (a) y la (b) hay un período en el que hay que liquidar las malas inversiones a la que la expectativa de ganancias, que ahora ya se ven exageradas, dio lugar. La burbuja tiene que «explotar», y esto generará virulentas reacciones contractivas en la economía que se contagiarán a través, principalmente, de la contracción del crédito y de la caída de los precios de los activos. La alimentación monetaria exógena de estas burbujas, por parte de una política monetaria expansiva del crédito dirigida por el banco central, no hace sino agravar la situación y retrasar el necesario reajuste de los precios y las rentabilidades de las inversiones.

${ }^{10}$ Hopkins et al. (2007).

11 Un episodio reciente de esto han sido las llamadas «.com». No se trata de un fenómeno nuevo: lo mismo ocurrió con la especulación de los canales y de los ferrocarriles en Inglaterra en el siglo XIX, etc. 
La conexión entre los procesos reales y financieros parece ahora más clara. Por supuesto no ha faltado quien ve en el «ciclo de los negocios» una consecuencia inevitable de las características del sistema financiero moderno: un sistema bancario de reserva fraccionaria, con un patrón monetario puramente fiduciario y un banco central que interfiere en la esfera de lo monetario. Tal es el caso de Rothbard (1972), para quien el paso de una fase expansiva a una contractiva se debe a un cluster de errores empresariales inducidos por una mala política monetaria (inflacionista), consecuencia de la actividad inflacionista de los propios bancos y de los gobiernos, que son comportamientos que afectan a los precios relativos y desvirtúan la información del mercado y los instrumentos de cálculo de los que se sirven los empresarios: el dinero y los precios.

En todo caso, entendemos que el papel de las expectativas de los diferentes agentes vinculadas a la aparición de oleadas de innovaciones es fundamental y no está suficientemente tratado en estas explicaciones exclusivamente monetarias de las crisis. A continuación se muestra cómo las características del sistema financiero actual interfieren en estos procesos agravando aún más las consecuencias de las posibles contracciones que se producen en su seno.

\section{LA CRISIS ACTUAL Y EL FUNCIONAMIENTO DEL SISTEMA FINANCIERO}

\subsection{Las características de un sistema monetario elástico}

Los procesos de innovación asumidos por los empresarios requieren necesariamente de un soporte financiero temporal para su desarrollo, especialmente en las primeras etapas del nacimiento de las empresas. De hecho, en ausencia de medios propios suficientes para su financiación, la persona que empieza una nueva actividad empresarial tiene la expectativa de poder recibir la cobertura financiera para cubrir un primer período de actividad, en que habitualmente los ingresos esperados no van a ser suficientes para hacer frente a los gastos en que se incurren en el lanzamiento de la nueva actividad. En esta fase inicial del desarrollo empresarial la función de los intermediarios financieros es casi determinante; la existencia o no de una banca dispuesta a la asunción (al menos parcial) del riesgo asociado a este tipo de operaciones, así como de un sistema de creación de liquidez flexible, pueden hacer que inversiones realmente innovadoras tengan alguna posibilidad de éxito, o bien no puedan llevarse a la práctica. No vamos a estudiar aquí la política de riesgos de las entidades bancarias sino que nos limitaremos 
a estudiar las características de los sistemas actuales de gobierno de la emisión de liquidez en nuestras economías y su incidencia en el movimiento económico ${ }^{12}$.

De acuerdo con las características del sistema bancario actual, basado en la reserva fraccionaria y en la existencia de un prestamista de última instancia en un patrón fiduciario, las entidades financieras pueden disponer de la mayor parte de los fondos de sus depositantes (ahorro de la economía) para la concesión de crédito a empresas y particulares sin asumir especiales riesgos, dentro de unos límites técnicamente prudenciales. Únicamente han de mantener un porcentaje de los depósitos recibidos en caja para atender a las retiradas de efectivo de sus clientes (coeficiente de caja o de reservas). A partir de ese ahorro depositado, las entidades financieras pueden crear y canalizar nuevos recursos concediendo créditos a los empresarios demandantes de financiación por un volumen total muy superior ${ }^{13}$ a los depósitos mantenidos en caja (propia y del banco central).

Esa multiplicación financiera del ahorro inicialmente disponible en la sociedad permite aumentar las posibilidades de financiación de la economía y, de hecho, abre paso a una mayor especialización de las entidades financieras. Ello se refleja en la existencia de un mercado amplio de servicios de intermediación financiera que guarda relación con el grado de riesgo que se esté dispuesto a asumir y con la expectativa de beneficio que se espera recibir en cada caso. Esta diversidad y especialización financieras permite la posibilidad de financiación de muchos proyectos innovadores que de otra forma no habrían podido ni siquiera comenzar a andar.

Ahora bien, esta forma de generación de liquidez también está expuesta a un mayor nivel general de riesgo y, por tanto, lleva asociada un componente de inestabilidad. Por un lado, es un sistema de generación de crédito que no está, en primera instancia, respaldado por una cantidad equivalente de ahorro o de depósitos en caja. Sólo a partir de una cantidad dada de recursos, que actúa como base de valor de las operaciones financieras, las entidades de crédito expanden sus activos por un valor total $k$ veces superior al de su pasivo (siendo $k$ el valor del multiplicador bancario); lo que se conoce como la expansión del crédito mediante el «apalancamiento financiero». Por otro lado, la utilización del sistema de reserva fraccionaria introduce necesariamente un desequilibrio temporal entre el plazo en que toma prestado el banco de los ahorradores (en su mayo-

12 Otros sistemas son, al menos teóricamente, posibles (véase Hayek (1978) y Selgin (1988)); pero para la interpretación de la crisis actual, vamos a considerar el sistema efectivamente vigente.

13 Esta capacidad para crear créditos está limitada por el valor del coeficiente de reservas y por la mayor o menor preferencia del público por los billetes y monedas de curso legal en lugar de depósitos bancarios. 
ría, a corto plazo) y al que presta a los demandantes de crédito (a medio y largo plazo). Aparecen así dos factores potenciales de desequilibrio en la gestión de este sistema de creación de liquidez, uno en cuanto a la enorme cantidad de créditos que se pueden crear ex novo dentro de unos límites muy amplios y otro en cuanto a la necesidad de conciliar la gestión de las partidas de un balance «temporalmente desequilibrado».

A todo ello hemos de añadir un factor de inestabilidad no menos importante, que consiste en que este sistema de creación de liquidez por los intermediarios financieros está basado en última instancia en un sistema monetario fiduciario puro. No es sólo que las entidades financieras puedan crear autónomamente y ex novo medios de pago, sino que todas las emisiones de liquidez descansan en un patrón monetario no respaldado en última instancia por el valor de ningún activo, real o financiero. Por tanto, es un sistema de creación autónoma (y plural por el número de oferentes involucrados) de medios de pago por parte de las entidades financieras dentro de un patrón monetario, que gobierna el banco central, de naturaleza plenamente fiduciaria. Esto hace que sea, al menos $a$ priori, un sistema de creación de liquidez técnicamente ilimitado y, por tanto, potencialmente inestable; lo que crea la necesidad de imponer límites (reglas) a la creación monetaria, tanto la del banco central como la del resto de entidades financieras.

La propia justificación de la existencia de un banco central, como banco de referencia de un «club» de bancos comerciales asociados a él, descansa en su papel como institución que provee servicios financieros que puedan reducir el nivel de riesgo con el que opera este sistema de creación de medios de pago (Goodhart, 1988). Entre ellos destacan la provisión de un patrón monetario estable, que sirva de base de valor para el resto de emisiones del sistema bancario, y la provisión regular y extraordinaria de crédito a las entidades asociadas a dicho $\left\langle\right.$ club» ${ }^{14}$.

En esta parte del trabajo nos ocuparemos de señalar algunas de las características de las reglas que se han establecido para limitar la creación de liquidez de los bancos centrales para, a continuación, poder estudiar la contribución que ha podido tener el crecimiento monetario resultante de su aplicación en la génesis y desarrollo de la di-

14 De hecho, sólo pueden emitir pasivos líquidos aquellos bancos admitidos por el banco central, a los que como banco que esencialmente es, presta liquidez y, como responsable último del buen funcionamiento del sistema de pagos, supervisa e inspecciona su política de préstamos y de inversiones, así como provee de financiación en casos de necesidad extraordinaria. Por tanto, interesa a todos los miembros de este «club» de emisión de medios de pago limitar la capacidad de emisión de liquidez de sus miembros; lo que para el banco central implica la adopción de una regla de emisión que mantenga el poder de compra del patrón de pagos de la economía. 
námica económica; y, en particular, en la explicación de una crisis económica como la actual.

\subsection{Reglas de los bancos centrales: ¿estabilización de precios?}

Especialmente desde la década de los 90 del siglo pasado, ha habido una tendencia generalizada en favor de la aplicación de un cierto tipo de reglas de gobierno de la política monetaria basadas en la llamada estabilización de precios $^{15}$; reglas que, en la práctica, persiguen la estabilización de la inflación en torno a una tasa de variación ligeramente positiva de un índice de precios de consumo (IPC). De acuerdo con este criterio de emisión, el banco central debe corregir toda desviación de la inflación respecto a la tasa objetivo elegida en cada caso; lo que, en los períodos de caída de los precios, ya sea una desinflación o una deflación, implica la fijación institucional de un límite inferior a la bajada de los precios. Como veremos, hay un tipo de bajadas de los precios que precisamente tiene lugar como elemento esencial (y esperado) de la dinámica que provoca una mejora de la productividad de una economía en crecimiento.

Con el fin de evitar el sesgo de sobre-medición de la inflación de los IPCs convencionales y guiados por el deseo de evitar toda deflación ${ }^{16}$ (véase BCE, 1999; Wynne 2009), los bancos centrales suelen concretar el objetivo de estabilidad de precios en la consecución de una tasa de variación interanual del IPC del 1-2\%. De esta manera, se fija un límite inferior a toda posible desinflación que pueda tener lugar en los mercados; lo que implicaría aumentar la oferta de liquidez para impedir que los precios caigan por debajo de ese límite durante una etapa expansiva de la economía, como resultado (natural y esperado) de aumentos de la productividad. Como consecuencia de la aplicación de este criterio de estabilización de la inflación, el banco central crearía liquidez para evitar la caída (esperada) de los precios resultado de una economía en crecimiento

15 Su propuesta y aplicación no son novedosas; puede encontrarse una crítica a sus efectos en los escritos monetarios de alguno de los exponentes más representativos de la escuela austriaca (Hayek, 1928).

16 Se adopta así una política monetaria intencionadamente asimétrica, dado que permite cierto margen de inflación y no así de deflación, independientemente del tipo que sea. Esta asimetría suele justificarse por la existencia de mayores costes asociados a la deflación, dado que se supone unida a episodios de recesión económica e inestabilidad financiera (véanse Burdekin y Sicklos, 2004 y BCE, 2003). 
(mayor oferta de bienes y servicios) en mercados abiertos; lo que distorsiona la información de los precios del mercado y, asimismo, aumenta la oferta de fondos prestables sobre bases no reales. Ambos elementos afectan a la dinámica empresarial y pueden fomentar la toma de decisiones de inversión erróneas sobre la base de expectativas y precios artificialmente sostenidos «monetariamente».

Esta prevención contra toda deflación vigente en nuestros días responde al recuerdo de los efectos asociados a un tipo particular de deflación -la de los años 30 del siglo pasado- que vino acompañada por una restricción masiva del crédito, una caída desconocida hasta entonces de la actividad y el empleo y un deterioro general de las expectativas. Ahora bien, a lo largo de la historia tenemos varios ejemplos de la existencia de varios tipos de deflaciones, con efectos muy distintos entre sí (véanse las registradas bajo patrones metálicos en el siglo xıx en Bordo y Filardo, 2004; Capie y Wood, 2004 y Castañeda, 2007). De ello podemos deducir la existencia de varios tipos de deflaciones que, para el desarrollo de una política monetaria guiada por la estabilidad monetaria a largo plazo, requieren distintas respuestas del banco central: en concreto, no siempre es deseable detener toda bajada de los precios inflando el mercado de liquidez (Castañeda, 2003).

\section{A. «Expansión económica, caídas de precios y respuesta inflacionista de las reglas actuales de política monetaria»}

Para entender la diversidad de los episodios de deflación, y en general de los distintos tipos de caídas de los precios, hemos de diferenciar entre las causas que conducen a variaciones del nivel de precios y las que únicamente provocan variaciones de unos precios en relación con otros. Las variaciones de la liquidez en circulación son las que explican las variaciones del nivel de precios y, por tanto, los fenómenos de inflación o deflación. Adoptando la denominación empleada por Hayek (1928), son las variaciones «por el lado del dinero» las que afectan al nivel general de los precios. Siguiendo la tradición monetaria iniciada por los miembros de la Escuela de Salamanca, continuada por los monetaristas del siglo pasado, se acepta como regla general que tanto la deflación como la inflación son fenómenos estrictamente monetarios a largo plazo. Por su parte, hay factores de origen real, como variaciones de la productividad en los distintos sectores y empresas, o el grado de apertura de los mercados, que explican cambios de los precios relativos entre los distintos bienes y servicios. Ahora bien, por la propia construcción de un verdadero índice general de precios, y en ausencia de variaciones monetarias, dichos cambios en los precios 
relativos no provocarán una variación del nivel de los precios. Siguiendo de nuevo la denominación de Hayek (1928), son ahora factores reales o «por el lado de los bienes» los que explican la variación de los precios relativos, pero no fenómenos como la inflación o la deflación.

Por último, hay conexiones entre las variaciones de precios relativos causadas por factores reales y las variaciones finales (monetarias) del nivel de precios. Siguiendo a Bordo y Schwartz (1979), hay un tipo de deflaciones que sí pueden resultar de aumentos de la productividad. En ausencia de variaciones monetarias, y ésta es una condición determinante, las mejoras de la productividad van acompañadas por el aumento de la oferta de bienes y servicios en los mercados, lo que en presencia de mercados abiertos provocará una tendencia a la baja de los precios a lo largo del tiempo. Es precisamente este tipo de caídas de los precios (de origen real), asociadas a un aumento de la oferta productiva, el que ha tenido lugar en los mercados mundiales durante la última etapa expansiva de la economía; que ha estado caracterizada por una caída de los precios a nivel mundial, resultado de un fuerte crecimiento de la producción mundial de bienes y servicios, así como por la globalización de los mercados de bienes y financieros.

Lo relevante para poder entender uno de los factores principales que ha acabado en una fuerte inestabilidad financiera en el verano de 2007 es analizar la reacción que ha tenido la política monetaria ante este tipo de caídas de los precios durante una etapa inequívocamente expansiva de la actividad. La respuesta de un banco central guiado por la estabilidad monetaria debe ser distinta de la que implicaría el desarrollo de reglas de estabilización de los precios en torno a un valor reducido de inflación (Castañeda, 2007). Si el banco central está guiado por un criterio de estabilidad monetaria, no tiene necesariamente que aumentar la liquidez para evitar toda deflación. Por estabilidad monetaria entendemos un criterio de gobierno de la liquidez que permita un crecimiento de los medios de pago compatible con la estabilidad del valor de compra de la moneda y la estabilidad del mercado financiero a largo plazo. Como veremos a continuación, guiados por reglas de estabilización de la inflación sesgadas contra toda deflación, así como por estrategias monetarias que han subestimado el crecimiento monetario registrado, los bancos centrales han permitido en general un aumento continuado (e insostenible) de la liquidez de la economía; lo que las convierte en reglas creadoras de un exceso de liquidez durante las etapas expansivas con efectos auténticamente desestabilizadores a largo plazo. 


\section{B. «Aplicación de estrategias monetarias y nuevos modelos macroeconómicos "no monetarios"»}

El espectacular crecimiento monetario registrado durante la etapa expansiva del ciclo que hemos vivido hasta verano de 2007 (véase gráfico 2) encuentra entre sus principales causas: (1) el desarrollo de reglas monetarias que no han respondido correctamente a la caída de los precios que tenía lugar en los mercados, que han resultado en políticas estrictamente inflacionistas e insostenibles a largo plazo; y (2) el diseño de estrategias monetarias que toman como referencia la «nueva» explicación de la inflación del llamado Nuevo Modelo Keynesiano (véanse Clarida et. al., 1999 y Galí, 2008), basado en los modelos de ciclo real a los que se añaden rigideces en la determinación de los precios nominales (incluidos los salarios).

\section{Modelos de referencia «inflacionistas»}

De acuerdo con este tipo de modelos, que son los de referencia en la academia e incluso en la práctica de muchos bancos centrales ${ }^{17}$, la inflación depende fundamentalmente de las expectativas de inflación y, a corto plazo, de las desviaciones de la actividad respecto de su tasa de crecimiento de equilibrio (el «output gap»). El modelo suele recogerse de manera abreviada a través de tres ecuaciones: (1) una nueva curva IS con expectativas, donde la actividad depende (negativamente) del tipo de interés real; (2) una nueva curva de Phillips neokeynesiana, donde la inflación depende de las expectativas de inflación así como del valor actual del «output gap»; y (3) una ecuación de cierre que se refiere a la determinación de los tipos de interés nominales por parte del banco central. Siguiendo este modelo, el tipo de interés de mercado óptimo ${ }^{18}$ es el que sigue al valor del tipo de

${ }^{17}$ Es necesario precisar que el modo de decidir la política monetaria en la práctica no sigue miméticamente estos modelos, ni los tipos de interés óptimos derivados de ellos (véase King, 2005). Estos modelos no recogen factores monetarios en la explicación de la inflación, y su aplicación directa implicaría un grado de conocimiento del modelo macroeconómico que dista mucho de la realidad en que operan los bancos centrales. Paddoa-Schioppa (2005) e Issing (2008) son ejemplos de críticas de los gestores de la política monetaria a este nuevo tipo de modelos.

${ }^{18}$ En esta literatura se entiende por óptimo el tipo de interés resultado de maximizar una función objetivo del banco central sujeto a las restricciones del modelo utilizado. 
interés natural o de equilibrio a largo plazo, y corrige las desviaciones esperadas tanto de la inflación respecto de su valor objetivo como del «output gap».

Se trata de un tipo de modelos denominado endógenos que consideran explícitamente que los cambios en las preferencias y tecnologías son exógenas. Los agentes deciden sobre la base de expectativas racionales que no consideran situaciones de incertidumbre en el sentido de Knight (1921), precisamente el tipo de situación típica que se produce en un contexto de fuerte cambio tecnológico.

¿Qué tipo de implicaciones tiene este modelo para la definición de «reglas monetarias óptimas»? En una economía con precios rígidos a corto plazo, el banco central, al decidir los tipos nominales, puede afectar a la producción y al empleo durante un cierto tiempo influyendo en los tipos de interés reales; lo que a su vez, por la propia dinámica del modelo, le permite influir en la tasa de inflación. Como implicación de política económica podemos destacar que un banco central estabilizador de la inflación tiene dos herramientas principales para conseguir su objetivo de inflación: (1) la elección de una estrategia monetaria reglada y creíble que logre fijar las expectativas de los agentes de acuerdo con sus objetivos y (2), una vez fijadas las expectativas de inflación, puede elegir el valor del tipo de interés nominal que anule el «output gap». Por tanto, de acuerdo con este modelo, la inflación se explica enteramente por la evolución de las expectativas y por factores reales a corto plazo; y el banco central tendría capacidad para influir en dichos factores para conseguir la tasa de inflación deseada.

Como consecuencia de ello, una vez fijado por el banco central un nivel de inflación óptimo, toda desinflación, y más aún si es deflación, se considera como un resultado subóptimo, cuando es precisamente ésta una bajada de los precios asociada a aumentos de la productividad que resultaría (naturalmente) de un mercado más competitivo. Por el contrario, este tipo de modelos prescribirían una reducción del tipo de interés nominal que lograra aumentar tanto las expectativas de inflación del mercado como la actividad a corto plazo y, con ello, reducir su desviación respecto a la producción de equilibrio. De nuevo, en un contexto de aumento de la productividad propio de una etapa expansiva, esta política monetaria conduciría a un aumento de los precios, dado que, en un contexto de expectativas de caídas de los precios por debajo del objetivo del banco central, este modelo prescribe el aumento de la cantidad de medios de pago de la economía y, por tanto, la creación de inflación ${ }^{19}$ por parte del banco central.

19 Decimos que se crea inflación (estrictamente monetaria) a pesar de que ello no se refleje siempre en aumentos de los IPCs, ya que se inyecta liquidez para frenar la caída de los precios que de otra forma tendría lugar en la etapa de crecimiento de la oferta productiva. 
Es decir, ni rastro de las (verdaderas) expectativas que forman los agentes en mercados muy dinámicos, que son precisamente los que están siendo afectados e inducen constante cambio técnico e institucional (cambio estructural).

\section{Estrategias de decisión «no monetarias»}

Asimismo, los partidarios de este tipo de modelos han defendido un cambio de las estrategias de los bancos centrales en la línea de abandonar las variables monetarias como guías de la política monetaria, y sustituirlas por nuevas estrategias que toman esos modelos y sus proyecciones como bases de la política monetaria (Svensson, 1999). Por tanto, ha habido también factores relacionados con la estrategia elegida por los bancos centrales que, por el menor peso decisorio asignado al crecimiento registrado de la liquidez como indicador de la estabilidad monetaria a medio y largo plazo, han permitido un exceso de crecimiento de los medios de pago durante la última fase de expansión de la economía. Este cambio de estrategia se ha reflejado fundamentalmente en dos áreas.

Por un lado, desde finales de la década de los 90, siguiendo a un cambio de sus estatutos que les otorgaron mayor transparencia e independencia operativa, muchos bancos centrales adoptaron un tipo de estrategia monetaria donde los agregados monetarios no tienen una función decisoria expresa. Este tipo de estrategia, llamada inflation targeting ${ }^{20}$, se basa fundamentalmente en el anuncio por parte del banco central de sus expectativas de evolución de la inflación a medio plazo (1-2 años); de modo que si las previsiones anunciadas difieren del objetivo de inflación del banco central podemos prever una variación del tipo de interés nominal en el sentido deseado en cada caso. La generación de estas previsiones de inflación es determinante en el desarrollo de la política monetaria; y son en general los nuevos modelos neokeynesianos antes señalados los utilizados como referencia para prever la tasa de inflación a corto plazo. Con ello, además de las expectativas de inflación, la estimación de una variable no observable como el «output gap» ha pasado a ser un elemento central de las previsiones ${ }^{21}$.

${ }^{20}$ Este ha sido el caso del Banco de Inglaterra, el Banco de Suecia, el Banco de Finlandia, el Banco de Reserva de Nueva Zelanda o el Banco de Canadá, entre muchos otros. Véase también King (2002) para un estudio de la función del dinero en este tipo de estrategias.

${ }^{21}$ Véase la nota 24 sobre los efectos desestabilizadores a que pueden conducir las reglas monetarias basadas en este tipo de variables. 
Por otro lado, el indicador elegido para medir la inflación suele ser el IPC. Por su propia definición, estos índices no son buenos indicadores de la evolución del poder de compra de la moneda. De hecho, el significativo crecimiento monetario registrado en los últimos años se ha trasladado a aumentos de los precios de los activos reales (particularmente, la inversión residencial) y financieros; y, sin embargo, el IPC ha permanecido en niveles bastante bajos ${ }^{22}$ y estables. Ello puede haberse debido a razones técnicas relacionadas con las características de este tipo de índices, así como económicas. En cuanto a las primeras, por su propia construcción, son índices parciales y sesgados (Cechetti y Groshen, 2001), que no recogen de manera correcta las variaciones de las condiciones monetarias de la economía, así como la evolución de la productividad de la misma. Puede haber aumentos de la productividad, ligados por ejemplo a innovaciones, en las etapas expansivas de la economía que, dependiendo del sector en que se generen, no se reflejen ni con la intensidad ni en el tiempo debidos en los IPCs. En cuanto a las razones económicas, la masiva incorporación a los mercados mundiales de economías (emergentes) como China e India, con una enorme capacidad de producción a precios reducidos, ha permitido absorber el crecimiento de la demanda de bienes y servicios de consumo final incluso con una clara caída de la inflación.

Todo ello ha creado una apariencia de estabilidad de los precios durante la última etapa expansiva que escondía una verdadera sobre-emisión de liquidez, que sí se reflejaba en una subida de los precios de los activos creada monetariamente; lo que ayudaba a mantener y promover en los empresarios expectativas artificiales acerca del crecimiento de los precios. Dicha apariencia de estabilidad de precios ha permitido tipos de interés de mercado históricamente bajos que han promovido un espectacular crecimiento de inversiones basadas en información distorsionada, y un aumento paralelo de la liquidez cuyos efectos no se han tenido suficientemente en cuenta.

22 La aplicación de la tesis monetarista anticipa que, ante un aumento de medios de pago, los agentes intentarán desprenderse del exceso no deseado de liquidez aumentando su demanda de activos reales, financieros, así como finalmente la de bienes y servicios. Ello resultará en un aumento de los precios de dichos activos y de los bienes y servicios (véase una explicación detallada en Congdon, 2005). 


\section{C. «La enseñanza monetarista en relación con la crisis reciente: medidas del excesivo crecimiento monetario»}

El BCE, a diferencia de otros bancos centrales, incluye expresamente el llamado «análisis monetario» (o primer pilar) como uno de sus elementos de decisión; a pesar de ello, ha desarrollado también una política monetaria que ha permitido un crecimiento de la liquidez en el área euro excesivo y, con el paso del tiempo, insostenible. Como veremos a continuación, el crecimiento de la oferta monetaria $\left(\mathrm{M}_{3}\right)$ ha sido muy superior a la propia tasa de referencia publicada por el BCE hasta 2003 (4.5\% interanual) ${ }^{23}$, así como a la tasa que resultaría de aplicar algunas reglas monetarias compatibles con la estabilidad de precios: ya sea en términos del tipo de interés nominal, como la llamada regla de Taylor (Taylor, 1993), ya sea en términos del crecimiento de un agregado monetario amplio, como la «regla del k \%» de Friedman o la regla de renta nominal de McCallum (1987).

Regla de Taylor con expectativas: regla de tipo de interés anti-inflacionista

¿Cuál habría sido el resultado de la aplicación en el área euro de la política prescrita por una regla de Taylor con expectativas (Clarida et. al., 1998)? Las funciones de reacción de este tipo pueden ser herramientas útiles para la explicación ex post ${ }^{24}$ de una política monetaria comprometida con la estabilidad de precios. De acuerdo con ellas, el tipo de interés de mercado debe guiarse por un componente de largo plazo, como es el tipo de interés natural o de equilibrio de la economía y otro de corto plazo, como es la corrección por anticipado de las desviaciones esperadas (E) tanto de la inflación respecto de su tasa objetivo como

23 Véase BCE (1999).

${ }^{24}$ Dadas las importantes limitaciones de este tipo de funciones de reacción como aproximación al modo en que se decide la política monetaria en la práctica (véase Orphanides, 2000), es importante señalar que las aplicamos aquí sólo como herramientas de explicación de la política monetaria registrada y no como si fueran medios efectivos de prescripción y conducción de la política monetaria. Son funciones de reacción que requieren de la estimación de variables no directamente observables (véase Leijonhufvud, 2007), expuestas a métodos de estimación controvertidos o, al menos, sobre los que no hay un consenso definitivo, que pueden conducir a la estimación de valores erróneos de las variables de decisión y, con ello, a la prescripción de una incorrecta política monetaria. Asimismo, hay muchos elementos y variables de decisión que no están presentes en esta sencilla formulación. 
del «output gap». De acuerdo con su parametrización convencional (véanse Taylor, 1993 y 1999), un banco central antiinflacionista, una vez fijado el valor del tipo de interés nominal de mercado $\left(i_{\text {nom }}\right)$ en línea con la evolución esperada del tipo de interés natural de la economía $\left(i_{\text {nat }}\right)$, reaccionaría con variaciones más que proporcionales del $i_{\text {nom }}$ ante desviaciones esperadas de la inflación de su tasa objetivo (coeficiente de reacción $g_{l}$ igual a 1.5) y con variaciones menos que proporcionales ante variaciones esperadas del «output gap» (coeficiente de reacción $g_{2}$ igual a 0.5 ). Con estos coeficientes de reacción y los datos disponibles desde el inicio de la política monetaria única en enero de 1999 hasta el comienzo de la crisis financiera en el verano de 2007, compararemos la evolución del tipo de interés nominal registrado con el que habría resultado de aplicar la siguiente función de reacción:

$$
i_{\text {nom }, t}=i_{n a t}^{*}+g_{1} E\left[\left(\Pi_{t+i}-\Pi^{*}\right)_{t}+g_{2} E\left[\left(\mathrm{Y}_{t+i}-\mathrm{Y}^{*}\right)_{t}\right]\right.
$$

Para su aplicación suponemos que la política monetaria se decide utilizando información esperada; en concreto, de acuerdo con el valor previsto ${ }^{25}$ en el trimestre siguiente del «output gap» y de las desviaciones de la inflación respecto del objetivo del BCE (inflación en el área euro menor si bien cercana al 2\% interanual). Para el cálculo del «output gap» y del tipo de interés natural, utilizaremos el filtro Hodrick-Prescott como aproximación a la tendencia de crecimiento de la economía a largo plazo. Nos queda la siguiente función de reacción:

$$
i_{n o m, t}=i_{n a t}^{*}+1.5 E\left[\left(\Pi_{t+i}-2 \%\right)_{t}+1.5 E\left[\left(\mathrm{Y}_{t+i}-\mathrm{Y}^{*}\right)_{t}\right]\right.
$$

Con las limitaciones señaladas (véase nota 24), utilizaremos los resultados de esta función de reacción como un medio para valorar ex post la evolución del tipo de interés de mercado en el área euro.

La comparación permite destacar que, especialmente desde 2003, el tipo de interés de mercado ha sido menor que el prescrito por una regla de estabilización de la inflación como la regla de Taylor utilizada (ec. 2). De acuerdo con ella, el menor tipo de interés de mercado fijado por el BCE puede haberse debido tanto a una subestimación del tipo de interés natural de la economía, como a una respuesta menos activa de lo necesaria ante desviaciones esperadas de la inflación y del «output gap».

25 En nuestro caso, únicamente por la mayor sencillez en la exposición de esta regla, bajo la hipótesis de previsión perfecta. 
Gráfico 1. Comparación del tipo de interés nominal del BCE con el resultante de aplicar la regla de Taylor. Fuente: Elaboración propia a partir de datos del BCE y del Banco de España

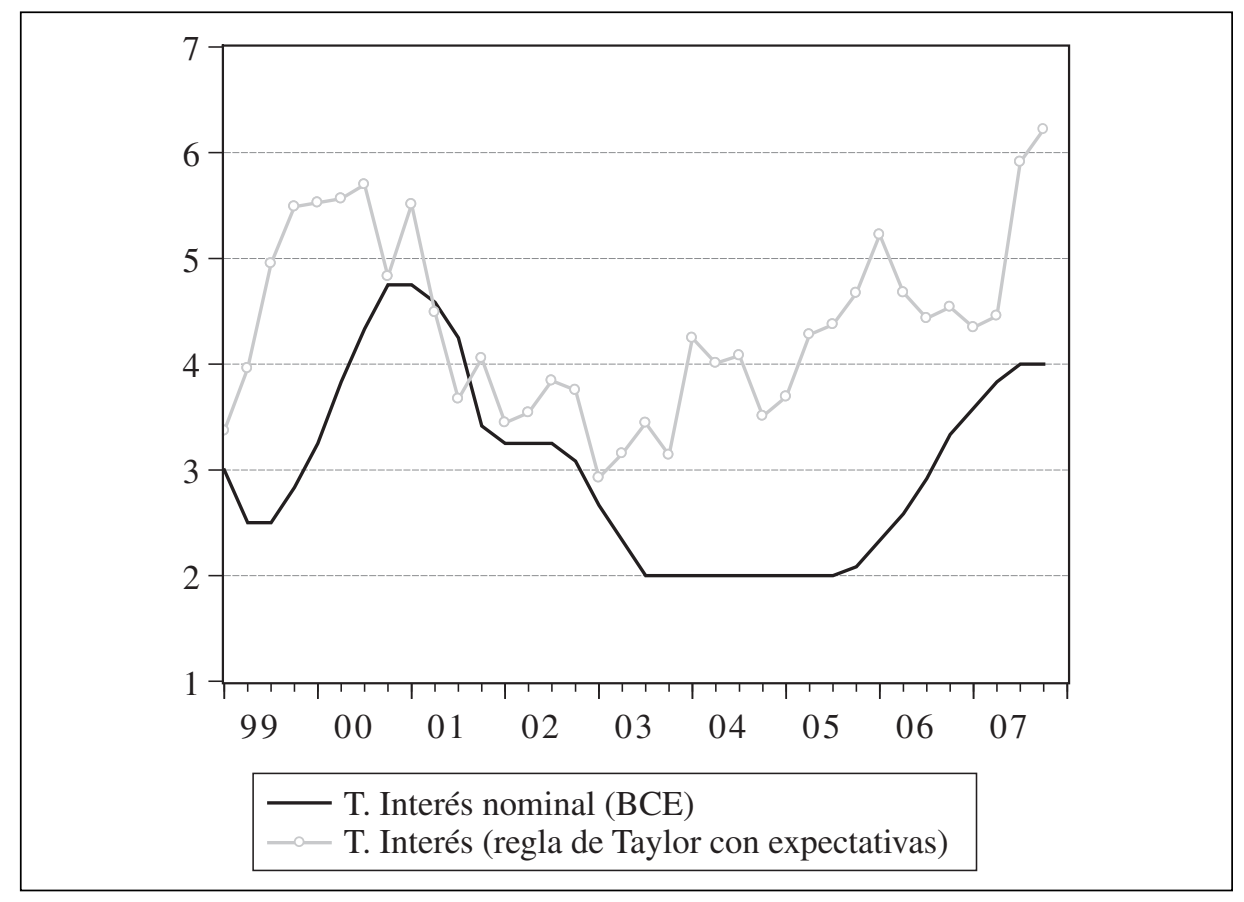

Comparación con reglas monetaristas

Los resultados anteriores son coherentes cuando comparamos el crecimiento monetario registrado en el área euro con el que resultaría de la aplicación de dos reglas monetaristas; reglas que, utilizando la ecuación cuantitativa del dinero, permiten identificar a grandes rasgos la tasa de crecimiento de la liquidez compatible con la estabilidad de precios a medio y largo plazo $^{26}$. Aunque la experiencia de la aplicación de la política

${ }^{26}$ En la actualidad los bancos centrales toman el tipo de interés y no un agregado monetario como instrumento de su política monetaria, por lo que en este ejercicio de evaluación del crecimiento monetario en el área euro hemos de tener presente que la evolución monetaria pasa a ser una variable endógena; y por tanto más volátil y dependiente de las decisiones tomadas sobre el valor del tipo de interés. 
monetaria del BCE es bastante reciente y aún no tenemos un número suficiente de años para un análisis empírico consistente, sí tenemos abundante experiencia histórica y fundamentos teóricos que demuestran la correlación entre el crecimiento monetario y el de la renta nominal y la inflación (Friedman, 2005) ${ }^{27}$.

Gráfico 2. Crecimiento monetario reciente en el área euro: comparación con reglas de estabilidad de precios. Fuente: Elaboración propia a partir de datos del BCE

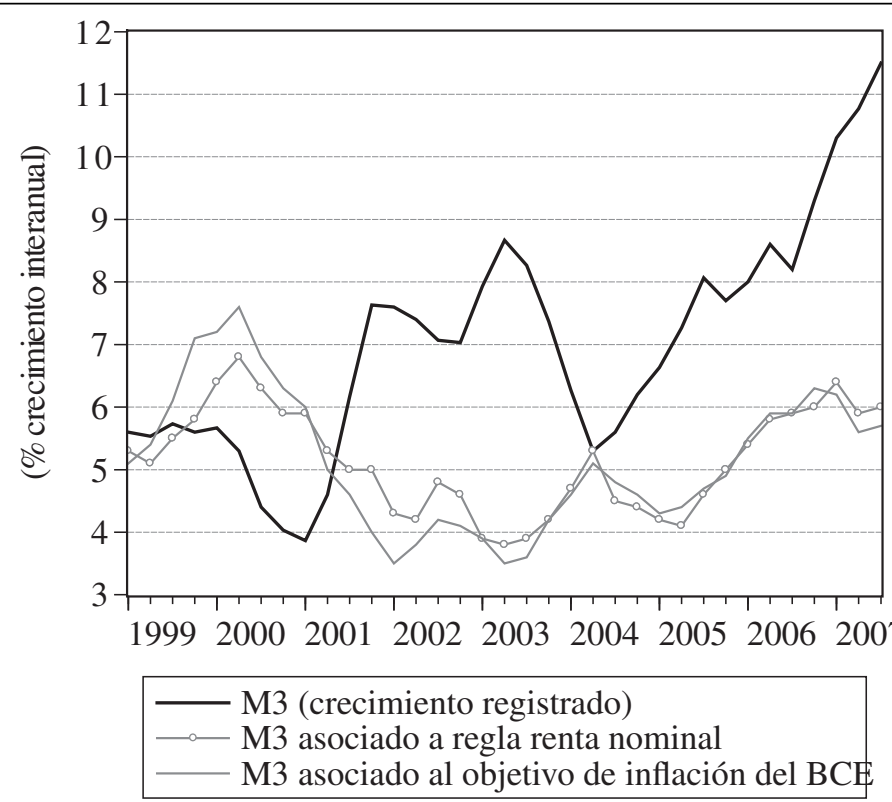

El crecimiento monetario compatible con el objetivo del BCE resultaría de la aplicación de la regla de Friedman, que no es más que una aplicación de la ecuación cuantitativa del dinero (véase ec. 3). Si fuera adoptada como regla de política monetaria, una vez descontada la caída estimada en la velocidad de circulación del dinero (-1\%), el BCE determinaría la tasa de crecimiento de liquidez que permitiera financiar el crecimiento de la economía a largo plazo (esto es, el crecimiento de la oferta de bienes y servicios, 2\%),

27 Véase Castañeda (2007) para un análisis similar de esta correlación durante la época del patrón oro en España y Congdon (2005) para el estudio monetarista de las últimas crisis registradas en el Reino Unido. 
asegurando un crecimiento de los precios no superior al $2 \%$ interanual. Se aprecia ahora más claramente que este tipo de reglas estabilizadoras de la inflación determinan la tasa de crecimiento monetario que, deliberadamente, impide una desinflación por debajo de la tasa objetivo anunciada, y mucho más cualquier tipo de deflación:

$$
\dot{\mathrm{M}}_{3}=\dot{\Pi}+\dot{\mathrm{Y}}_{\text {Tend }}-\dot{V}_{\text {Tend }}=2 \%+2 \%-(-1 \%)=5 \%
$$

Asimismo, si adoptamos un criterio de evolución de los agregados monetarios de acuerdo con la evolución de la renta nominal de la economía, a diferencia de la regla anterior, el crecimiento monetario se determina en función de la evolución del crecimiento de la producción a los precios esperados (ec. 4); esto es, sin fijar de antemano un objetivo de inflación ${ }^{28}$ :

$$
\dot{\mathrm{M}}_{3}=\left.\left(\dot{\mathrm{Y}}_{\text {Tend }}+\Pi\right)\right|^{\mathrm{E}}-\dot{V}_{\text {Tend }}
$$

\section{Conclusiones}

De acuerdo con la teoría monetaria y la evidencia empírica conocida, resulta difícil asumir que el exceso de liquidez generado en los últimos años no haya tenido los efectos previsibles, y comentados en este trabajo, sobre la distorsión de los precios y una incorrecta formación de las expectativas de los agentes en los distintos mercados. El tipo de interés fijado por el BCE ha registrado en los últimos diez años una media en torno al 3\%, lo que es un punto porcentual menor al prescrito en media por una regla de estabilización de la inflación en torno al objetivo del BCE como la regla de Taylor. Puede observarse en el gráfico 2 que el crecimiento monetario registrado en el área euro ha sido mucho mayor que el aconsejado por reglas guiadas por la estabilidad de la inflación a medio y largo plazo, como son las de Friedman y McCallum; así como respecto a la propia tasa de referencia para el crecimiento de $\mathrm{M}_{3}$ anunciada anualmente por el BCE desde 1999 hasta 2003.

Sin embargo, en nuestra opinión esto no es suficiente para explicar la sucesión de movimientos expansivos y contractivos típicos de las economías de mercado. Es nece-

${ }^{28}$ Con esta regla, en principio, sí se permitiría una bajada de los precios que acompañara a un aumento de la producción de bienes y servicios; lo que podría resultar en un crecimiento monetario menor. 
sario considerar el mecanismo de generación de expectativas vinculadas a los procesos de generación e introducción de innovaciones por parte del empresario. La dinámica descrita de sobre-emisión de liquidez en la esfera monetaria y financiera, en el contexto actual, no hace sino amplificar los errores empresariales, sobre todo si se asumen determinadas reglas de política monetaria (y fiscales) que son implícitamente expansivas. Amparadas en la consecución de una estabilidad de los precios mal entendida y definida, son reglas que no contribuyen a la estabilidad monetaria en el medio y largo plazo, lo que perjudica a la generación de expectativas correctas por parte de los agentes y al alargamiento artificial de las etapas expansivas. Por todo ello, el siguiente paso consistiría en elaborar un marco teórico mejorado, que integre en una única explicación ambas dinámicas (monetaria y real), y en el que la expansión del crédito no es sino la otra cara de la innovación. Si bien han existido muy notables intentos (muchos de ellos se han citado en el trabajo), aún no se ha llegado, a nuestro juicio, a una exposición definitiva.

\section{REFERENCIAS BIBLIOGRÁFICAS}

BCE (1999), «La estrategia monetaria del Eurosistema orientada hacia la estabilidad», Boletín Mensual del BCE, enero, págs. 45-57.

- (2003), «Resultados de la evaluación del BCE de su estrategia de política monetaria», Boletín Mensual del BCE, Junio, págs. 83-97.

Bordo, Michael y Filardo, Andrew (2004), «Deflation and Monetary Policy in a Historical Perspective: Remembering the Past or Being Condemned to Repeat It?» NBER Working Paper No. 10833, October 2004.

Bordo, Michael y Schwartz, Anna (1979), «Money and Prices in the Nineteenth Century: Was Thomas Tooke Right?», Carleton Economic Papers, 20.

Burdekin, Richard C. K. y Siklos, Pierre L. (eds.) (2004), Deflation: Current and Historical Perspectives, Cambridge, Cambridge University Press.

CapIe, Forrest y Wood, Geoffrey E. (2004), «Price Change, Financial Stability and the British Economy, 1870-1939», en Burdekin y Siklos (eds.), Deflation: Current and Historical Perspectives, Cambridge, Cambridge University Press, págs. 61-90.

Castañeda, Juan (2003), Propuesta de una regla de emisión para el BCE. De la estabilidad de precios a la estabilidad monetaria, Colección de tesis doctorales, Ed. UAM.

- (2007), ¿Puede haber deflaciones asociados a aumentos de la productividad? Análisis de la producción y los precios en España entre 1868 y 1914, Serie de Estudios de Historia Económica, Banco de España. 
Cecchetti, Stephen y Groshen, Erica (2001), «Understanding inflation. Implications for Monetary Policy», en P. Dreze (ed.), Advances in Macroeconomic Theory, Palgrave, págs. 113-135.

Clarida, Richard; Galí, Jordi y Gertler, Mark (1998), «Monetary Policy Rules in Practice. Some International Evidence», European Economic Review 42, págs. 1033-1067.

- (1999), «The Science of Monetary Policy: A New Keynesian Perspective», Journal of Economic Literature, vol. XXXVII, págs. 1661-1707.

Congdon, Tim (2005), Money and asset prices in boom and bust, Institute of Economic Affairs, Hobart Paper N. 152.

Dosi, Giovanni (1982), «Technological paradigms and technological trajectories. A suggested interpretation of the determinants and directions of technological change», Research Policy 11, págs. 147-162.

Dupriez, Léon-H. (1959), Philosophie des conjoctures économiques, Louvain et Paris, Institut de Recherches Economiques et Sociales de l'Université de Louvain, E. Nauwelaerts et Béatrice-Nauwelaerts.

Encinar, María Isabel y MuÑoz, Félix-Fernando (2006), «On novelty and economics: Schumpeter's paradox», Journal of Evolutionary Economics 16(3), págs. 255-277.

FisHer, Irving (1933), «The Debt-Deflation Theory of Great Depressions», Econometrica 1(4), págs. 337-357.

Freeman, Christopher y Louça, Francisco (2001), As time goes by. From the industrial revolution to the information revolution, Nueva York y Oxford, Oxford University Press.

FriedMAN, Milton (2005), «A natural experiment in monetary policy covering three episodes of growth and decline in the economy and the stock market», Journal of Economic Perspectives (19)4, págs. 145-150.

Friedman, Milton y Schwartz, Anna (1963), A Monetary History of the United States, 18671960, Princeton, Princeton University Press.

Galí, Jordi (2008), Monetary Policy, Inflation and the Business Cycle, Princeton University Press.

GARrison, Roger W. (2005), Tiempo y Dinero. La macroeconomía de la estructura del capital, Unión Editorial.

Goodhart, Charles (1988), The Evolution of Central Banks, Cambridge MA, The MIT Press.

HAYEK, Friedrich von (1928), «Intertemporal Price Equilibrium and Movements in the Value of Money», en R. McCloughry (ed.), Money, Capital and Fluctuations, Routledge and Keagan Paul (1984), págs. 71-117.

- (1931), Prices and Production, Londres, Routledge and Sons.

- (1978), Denationalisation of Money. An Analysis of the Theory and Practice of Concurrent Currencies, Londres, Institute of Economic Affairs.

Hicks, John R. (1967), «The Hayek Story», Critical Essays in Monetary Theory, Oxford, Clarendon Press, págs. 203-215. 
Hopkins, Michael M.; Martin, Paul A.; Nightingale, Paul; Kraft, Alison y Mahdi, Surya (2007), «The myth of the biotech revolution: An assessment of technological, clinical and organisational change», Research Policy 36, págs. 566-589.

Issing, Otmar (2008), The Birth of the Euro, Cambridge, Cambridge University Press.

KING, Mervyn (2002), «No money, no inflation-the role of money in the economy», Bank of England Quarterly Bulletin, Verano.

- (2005), «Monetary Policy: Practice ahead of Theory», Conferencia en Cass Business School (Mais Lecture), 17 de mayo, Londres.

Matsuyama, Kiminori (1999), «Growing though cycles», Econometrica 67(2), págs. 335-347.

McCallum, Bennett T. (1987), «The Case for Rules in the Conduct of Monetary Policy: A Concrete Example», Federal Reserve Bank of Richmond Economic Review 73(5), págs. 10-18.

Nelson, Richard y Winter, Sydney (1982), An Evolutionary Theory of Economic Change, Hardvard, Harvard University Press.

LeiJonhufvud, Axel (2007), «Monetary and financial stability», CEPR Policy Insight N14.

Lucas, Robert Jr. (1981), Studies in Business-cycle Theory, Oxford, Basil Blackwell.

Mises, Ludwig von (1924 [1997]), La teoría del dinero y del crédito, Madrid, Unión Editorial.

- (1949), Human action: A Treatise on Economics, New Haven, Yale University Press.

OrPHANIDES, Athanasios (2000), «Activist Stabilisation Policy and Inflation. The Taylor Rule in the 1970s», Board of Governors of the Federal Reserve System (Febrero).

Padoa-Schioppa, Tommaso (2004), The Euro and its Central Bank. Getting united after the union, Cambridge MA, The MIT Press.

PÉREZ, Carlota (2004), Revoluciones tecnológicas y capital financiero. La dinámica de las grandes burbujas financieras y las épocas de bonanza, México DF y Buenos Aires, Siglo XXI.

Rothbard, Murray N. (1972 [1963]), America's Great Depression, Nueva York, Richardson \& Snyder.

Schumpeter, Joseph A. (1934 [1983]), The theory of economic development. An inquiry into profits, capital, credit, interest, and the business cycle, Harvard, Transaction Publishers.

- (1939 [1989]), Business Cycles. A theoretical, historical and statistical analysis of the capitalist process, Philadelphia, Porcupine Press.

Selgin, George A. (1988), The Theory of Free Banking: Money Supply under Competitive note Issue, Totowa, NJ, Rowan \& Littlefield.

Shackle, George S. (1972), Epistemics and Economics, Cambridge, Cambridge University Press.

Sмiтh, Vera (1936 [1993]), Fundamentos de la banca central y de la libertad bancaria, AostaUnión Editorial.

Svensson Lars. E. O. (1999), «Monetary Policy Issues for the Eurosystem», International Macroeconomics, CEPR núm. 2197 (Agosto). 
TAYLOR, John B. (1993), Macroeconomic Policy in a World Economy. From Econometric Design to Practical Operation, Nueva York y Londres, Norton \& Company.

TAYLOR, John B. (1999), «A Historical Analysis of Monetary Policy Rules», en J. B. Taylor (ed.), Monetary Policy Rules, Chicago, NBER, págs. 319-347.

WynNe, Mark (2009), «How should central banks define price stability?», en D. Mayes y G. E.

Wood (eds.), Designing Central Banks, Abingdon, Routledge (en prensa). 